\title{
New Rewritings and Optimizations for Regular Path Queries
}

\author{
Gösta Grahne and Alex Thomo \\ Concordia University, \\ Montreal, Canada \\ \{grahne, thomo\}@cs.concordia.ca
}

\begin{abstract}
All the languages for querying semistructured data and the web use as an integral part regular expressions. Based on practical observations, finding the paths that satisfy those regular expressions is very expensive. In this paper, we introduce the "maximal partial rewritings" (MPR's) for regular path queries using views. The MPR's are always exact and more useful for the optimization of the regular path queries than other rewritings from previously known methods. We develop an algorithm for computing MPR's and prove, through a complexity theoretic analysis, that our algorithm is essentially optimal. Also, we present query answering algorithms that utilize exact partial rewritings for regular path queries and conjunctive regular path queries respectively.
\end{abstract}

\section{Introduction}

Semistructured data are self-describing collections, whose structure can naturally model irregularities that cannot be captured by relational or object-oriented data models [1. This kind of data is usually best formalized in terms of labeled graphs, where the graphs represent data found in many useful applications such as web information systems, XML data repositories, digital libraries, communication networks, and so on. Virtually, all the query languages for semi-structured data provide the possibility for the user to query the database through regular expressions. The design of query languages using regular path expressions, is based on the observation that many of the recursive queries that arise in practice amount to graph traversals. These queries are in essence graph patterns, and the answers to the query are subgraphs of the database that match the given pattern 17.7 , 34 .

For example, for answering a query containing in it the regular expression $\left({ }^{*} \cdot\right.$ article $) \cdot\left({ }_{-}^{*} \cdot\right.$ ref $\cdot_{-} *($ abiteboul + vianu $\left.)\right)$, one should find all the paths having at some point an edge labeled article, followed by any number of other edges, then by an edge ref, and finally by an edge labeled with abiteboul or vianu.

Based on practical observations, the most expensive part of answering queries on semistructured data is to find those graph patterns described by regular expressions. This is because a regular expression can describe arbitrarily long paths in the database, which means in turn an arbitrary number of physical accesses. Hence, it is clear that having a good optimizer for answering regular 
path queries is very important. This optimizer can be used for the broader classes of query languages for semistructured data, as we illustrate in the paper.

In semistructured data as well as in other data models, such as relational and "object oriented," the importance of utilizing views is well recognized [16. 315 18. Simply stated the problem is: Given a query $Q$ and a set of views $\left\{V_{1}, \ldots, V_{n}\right\}$, find a representation (rewriting) of $Q$ by means of the views and then answer the query on the basis of this representation.

The most notable methods for obtaining rewritings for regular path queries are by Calvanese et al in 356 . However, these methods rewrite -using viewsonly complete words of the query. But in practice, the cases in which we can infer from the views full words for the query, are very "ideal." The views can cover partial words that can be satisfactorily long for using them in optimization, but if they are not complete words, they are ignored by the above mentioned methods. It would however be desirable to have a partial rewriting in order to capture and utilize all the information provided by the views.

The problem of computing a partial rewriting is initially treated by Calvanese et at in [3]. There this problem is considered as an extension of the complete rewriting, enriching the set of the views with new elementary one-symbol views, chosen among the database relations (or symbols). The choice of the new elementary views is done in a brute force way, using a cost criterion depending on the application. However, there are cases when the algorithm of [3] for computing partial rewritings gives "too much" (in a sense to be made precise later). It essentially contains redundant un-rewritten (sub)words from the query.

In a previous paper [10, the present authors presented another algorithm for computing contained partial rewritings. Using that algorithm we avoid getting "too much," but unfortunately, the rewriting is not guaranteed to be exact, which diminishes its usability.

In this paper we will introduce the "maximal partial rewritings" (MPR's) for regular path queries using views. We will show that they don't give "too much," that they are always exact and more useful for the optimization of the regular path queries.

Then, we will present an algorithm that, when using exact partial rewritings, optimizes the evaluation of regular path queries to a database. We also explore the use of the partial rewritings for the optimization of the wider class of conjunctive regular path queries (CRPQ's). We introduce the "conjunctive exact partial rewritings" (CEPR's) and present an algorithm for utilizing them in CRPQ evaluation.

Finally, through a complexity theoretic analysis, we prove that our algorithm for computing the "maximal partial rewritings" is essentially optimal.

Due to space limitations, the proofs of some theorems and lemmas are omitted. These proofs appear in the full version of the paper [12].

\section{Background}

Semistructured databases. We consider a database to be an edge labeled graph. This graph model is typical in semistructured data, where the nodes of 
the database graph represent the objects and the edges represent the attributes of the objects, or relationships between the objects.

Formally, we assume that we have a universe of objects $D$ and a finite alphabet $\Delta$, called the database alphabet. Objects of $D$ will be denoted $a, b, c, a^{\prime}, b^{\prime}$, $\ldots, a_{1}, b_{2}, \ldots$, etc. Elements of $\Delta$ will be denoted $R, S, T, R^{\prime}, S^{\prime}, \ldots, R_{1}, S_{1}, \ldots$, etc.

A database $D B$ over $(D, \Delta)$ is a pair $(N, E)$, where $N \subseteq D$ is a set of nodes and $E \subseteq N \times \Delta \times N$ is a set of directed edges labeled with symbols from $\Delta$.

Queries and rewritings of queries using views. A regular path query $Q$ is a finite or infinite regular language over the alphabet $\Delta$. Let $Q$ be a regular path query, and $D B=(N, E)$ a database. Then the answer to $Q$ on $D B$ is defined as:

$$
\text { ans }(Q, D B)=\left\{(a, b) \in N^{2}: a \stackrel{W}{\longrightarrow} b \text { for some } W \in Q\right\} .
$$

Let $\mathbf{V}=\left\{V_{1}, \ldots, V_{n}\right\}$ be a set of view definitions with each $V_{i}$ being a finite or infinite regular language over $\Delta$. Associated with each view definition $V_{i}$ there is a view name $v_{i}$. We call the set $\Omega=\left\{v_{1}, \ldots, v_{n}\right\}$ the outer or view alphabet. For each $v_{i} \in \Omega$, we set $\operatorname{def}\left(v_{i}\right)=V_{i}$. The substitution def associates with each view name $v_{i}$, in the $\Omega$ alphabet, the language $V_{i}$. Also, we extend the substitution def to the $\Delta$ alphabet associating each symbol with itself. The substitution def is applied to words, languages, and regular expressions, over $\Omega \cup \Delta$ in the usual way (see e. g. [13]). Sometimes we need to refer to regular expressions representing the languages $Q$ and $V_{i}$. In order to simplify the notation, we will blur the distinction between the regular expressions and the languages that they represent.

The maximally contained rewriting, of a user query $Q$ using $\mathbf{V}\left(M C R_{\mathbf{V}}(Q)\right)$, is the language $Q^{\prime}$ over $\Omega$ that includes all the words $v_{i_{1}} \ldots v_{i_{k}} \in \Omega^{*}$, such that

$$
\operatorname{def}\left(v_{i_{1}} \ldots v_{i_{k}}\right) \subseteq Q 1
$$

The $M C R_{\mathbf{V}}(Q)$ can be empty even if the desired answer is not. Suppose, for example, that the query $Q$ is $Q=R_{1} \ldots R_{100}$ and we have available two views $V_{1}$ and $V_{2}$, where $V_{1}=R_{1} \ldots R_{49}$ and $V_{2}=R_{51} \ldots R_{100}$. It is easy to see that $M C R_{\mathbf{V}}(Q)$ is empty. However, depending on the application, a "partial rewriting" such as $v_{1} R_{50} v_{2}$ could be useful. In the next section we develop a formal algebraic framework for the partial rewritings. In sections [5] and [6] we demonstrate the usefulness of the partial rewritings in query optimization.

\section{Partial Rewritings}

Let $L$ be a language and $M$ an $\epsilon$-free language, both of them over some alphabet $\Delta$. Let $m$ be a symbol outside $\Delta$ and set $\operatorname{def}(m)=M$. A partial $M$-rewriting of

\footnotetext{
${ }^{1}$ It is easy to see that this definition of $M C R \mathbf{V}(Q)$ matches exactly the language of the output automaton from Theorem 1 in [3], which is the rewriting as defined there, with respect to the view alphabet.
} 
$L$ is a language $L^{\prime}$ over $\Delta \cup\{m\}$, such that $\operatorname{def}\left(L^{\prime}\right) \subseteq L$. The partial rewriting is said to be exact, if $\operatorname{def}\left(L^{\prime}\right)=L$. Since the (complete) rewritings, i.e. the ones that use only the $m$ symbol, are special cases of the partial rewritings, we will blur the distinction between them and call the partial rewritings just rewritings.

It is clear that there can be several $M$-rewritings of the same language $L$. In order to compare different rewritings, we introduce a partial order between the languages over $\Delta \cup\{m\}$. With this partial order we want to capture the intuition that the more subwords on the $\Delta$-alphabet that have been replaced by $m$ in a rewriting, the "bigger" (and "better") the rewriting.

Let $L_{1}$ and $L_{2}$ be languages over $\Delta \cup\{m\}$. We define $L_{1}$ to be $M$-smaller than $L_{2}$, denoted $L_{1} \leq_{M} L_{2}$, if it is possible to substitute by $m$ some (not necessarily all) occurrences of words over $M$ occurring as subwords in $L_{1}$, and obtain $L_{2}$ as a result.

Obviously $\leq_{M}$ is transitive and reflexive. It is not antisymmetric, as for instance $\{m R R, m m, R R R R\} \leq_{M}\{m m, R R R R\}$, and $\{m R R, m m, R R R R\}$ $\geq_{M}\{m m, R R R R\}$, when $M$ is for example $\{R R\}$. However, if we define $L_{1} \equiv_{M}$ $L_{2}$ iff $L_{1} \leq_{M} L_{2}$ and $L_{2} \leq_{M} L_{1}$, we will get a partial order on the equivalence classes.

Notably, we have that if a set $L^{\prime}$ is $\leq_{M}$-maximal, then its equivalence class is singleton. To show this, we first present the following theorem.

Theorem 1. Let $L^{\prime}$ be a language on $\Delta \cup\{m\}$. Then $L^{\prime}$ is $\leq_{M}$-maximal, if and only if, there does not exist a word $W \in L^{\prime}$, such that $W=W_{1} W_{2} W_{3}$, and $W_{2} \in M$.

Corollary 1. Let $L^{\prime}$ be a language on $\Delta \cup\{m\}$. If $L^{\prime}$ is $\leq_{M}$-maximal, then its $\equiv_{M}$-equivalence class is a singleton.

Now, consider this partial order restricted to the set of all the $M$-rewritings of a language $L$. We will denote the restricted partial order with $\leq_{M}^{L}$. Obviously, we are interested in the $\leq_{M}^{L}$-maximal $M$-rewritings of $L$. With a similar reasoning as before, we can prove the next theorem and corollary. The main difference is that we cannot now replace just any subword which is a word in $M$. Naturally, a word $W=W_{1} W_{2} W_{3}$, where $W_{2} \in M$ and $\operatorname{def}\left(W_{1} m W_{3}\right) \subseteq L$, is not yet an "optimal" word, and we call $W_{2}$ a subword eligible for replacement.

Theorem 2. Let $L^{\prime}$ be a rewriting of $L$ on $\Delta \cup\{m\}$. Then $L^{\prime}$ is $\leq_{M}^{L}$-maximal, if and only if, there does not exist a word $W \in L^{\prime}$, such that $W=W_{1} W_{2} W_{3}$, where $W_{2} \in M$, and def $\left(W_{1} m W_{3}\right) \subseteq L$.

Corollary 2. Let $L^{\prime}$ be a rewriting on $\Delta \cup\{m\}$. If $L^{\prime}$ is $\leq_{M}^{L}$-maximal, then its $\equiv_{M}^{L}$-equivalence class is a singleton.

Also, we require exactness for a rewriting. Only then a rewriting becomes useful for query optimization 411 .

A rewriting that is both $\leq_{M}^{L}$-maximal and exact is the union of all $\leq_{M^{-}}^{L}$ maximal $M$-rewritings of $L$. We call this rewriting the maximal partial $M$ rewriting of $L$, and denote it with $M P R_{M}(L)$. 
From all the above, we can see that the $M P R_{M}(L)$ is the set of all the words on $\Delta \cup\{m\}$ with no subword for potential "contained replacement."

Example 1. Let $M=\{R S R, S\}$, and

$$
L=\{R S R S, S R S R, R S R R S R, S S, S R S R S, S S S\}
$$

Then

$$
M P R_{M}(L)=\{m m, S m S\} .
$$

Here, $m m$ is obtained from $R S R S, S R S R, R S R R S R$, or $S S$. The word $S m S$ is obtained from $S R S R S$ or $S S S$. There are no more eligible subwords for replacement. For instance, replacing subwords in $\underline{S} m \underline{S}$ that belong to $M$, would violate the containment condition necessary for being a rewriting.

Formally, we have that:

Theorem 3. The rewriting $\operatorname{MPR}_{M}(L)$ is $\leq_{M}^{L}$-maximal and exact.

We can give a natural generalization of the definition of the maximal partial M-rewriting for the case when we like to replace subwords not from one language only, but from a finite set of languages (such as a finite set of view definitions). For this purpose, suppose we are given the $\epsilon$-free view languages $\mathbf{V}=\left\{V_{1}, \ldots, V_{n}\right\}$ and a target query language $Q$, all of them over the alphabet $\Delta$. Also, consider the view alphabet $\Omega=\left\{v_{1}, \ldots, v_{n}\right\}$, for which as defined in Section 2 , we have $\operatorname{def}\left(v_{i}\right)=V_{i}$, for $i \in[1, n]$. A partial $\mathbf{V}$-rewriting of $Q$ is a language $Q^{\prime}$ over $\Delta \cup \Omega$, such that $\operatorname{def}\left(Q^{\prime}\right) \subseteq Q$. The partial rewriting is said to be exact if $\operatorname{def}\left(Q^{\prime}\right)=Q$. Then, in order to compare the rewriting we define analogously the partial order $\leq \mathbf{v}$ on $(\Delta \cup \Omega)^{*}$ as follows.

Let $Q_{1}$ and $Q_{2}$ be languages over $\Delta \cup \Omega$. We define $Q_{1}$ to be $\mathbf{V}$-smaller than $Q_{2}$, denoted $Q_{1} \leq_{\mathbf{V}} Q_{2}$, if it is possible to substitute by $v_{i_{1}}, \ldots, v_{i_{k}}$ some (not necessarily all) occurrences of words over $V_{i_{1}}, \ldots, V_{i_{k}}$ respectively, occurring as subwords in $Q_{1}$, and obtain $Q_{2}$ as a result.

As before, we restrict this partial order to the set of all the $\mathbf{V}$-rewritings of a language $Q$. Similarly, we denote the restricted partial order with $\leq_{\mathbf{V}}^{Q}$. Finally, we define the maximal partial $\mathbf{V}$-rewriting of $Q$, denoted $M P R_{\mathbf{V}}(Q)$, to be the union of all $\leq_{\mathbf{V}}^{Q}$-maximal $\mathbf{V}$-rewritings $Q^{\prime}$ of $Q$. Clearly, as in Theorem 3 , we can show that the $M P R_{\mathbf{V}}(Q)$ is $\leq_{\mathbf{V}}^{Q}$-maximal and exact.

We will compare in the following the MPR's with the partial rewritings proposed in the literature. We begin with the partial rewriting of [3]. In that paper, candidate sub-alphabets $\Delta^{\prime} \subseteq \Delta$ are selected using some cost criteria, and then the symbols of $\Delta^{\prime}$ are considered as new elementary one-symbol views. Note that there are exponentially many candidate sub-alphabets. Each time, using the algorithm presented in [3], a rewriting is computed and after that its exactness is tested. However, the algorithm used, although very suitable for computing all the rewritings in the view alphabet $\Omega$, can compute "non-optimal" $\Delta \cup \Omega$ words when it is used for computing partial rewritings. As an example, consider the regular path query $Q=R_{1} R_{2}+R_{2}^{5}$ and two views $V_{1}=R_{1}$ and $V_{2}=R_{2}^{5}$. Then, 
the algorithm of [3] will give as partial rewriting $V_{1} R_{2}+V_{2}+R_{2}^{5}$, which has the redundant "non-optimal" word $R_{2}^{5}$. As a consequence, the partial rewriting of [3] is not always $\leq_{\mathbf{V}}^{Q}$-maximal. We call the partial rewriting of [3] the maximally contained partial rewriting or $M C P R_{\mathbf{V}}$ because it contains all the words $W$ over $\Delta^{\prime} \cup \Omega$, such that $\operatorname{def}(W) \subseteq Q$. Observe here that the maximality in this rewriting is with respect to the containment of $\Delta^{\prime} \cup \Omega$ words and not really to their optimality regarding the non-view symbols.

Now, let's consider the partial rewriting presented in [11]. This rewriting is the union of all $\leq \mathbf{v}$-maximal $\mathbf{V}$-rewritings of $Q$. By Theorem 1 we have that this is the set of all "mixed" words $W$ on the alphabet $\Omega \cup \Delta$ with no subword in $V_{1} \cup \cdots \cup V_{n}$, such that their substitution by def is contained in the query $Q$. We call this set exhaustive lower partial rewriting, or $\operatorname{ELP} R_{\mathbf{V}}(Q)$, because we replace subwords of $Q$ with view symbols thinking only about the optimality of words with regard to the non-view symbols, but not caring about the exactness of the rewriting. Clearly, since any $\leq_{\mathbf{v}}$-maximal rewriting is also $\leq_{\mathbf{V}}^{Q}$-maximal, the result is that we get always a $\leq_{\mathbf{V}}^{Q}$-maximal rewriting, but its exactness is not guaranteed.

Finally, $M P R_{\mathbf{V}}(Q)$ is a rewriting that satisfies both the optimality with regard to the non-view symbols and the exactness. Summarizing, and also including the maximally contained (complete) rewriting $M C R_{\mathrm{V}}$ in the comparison, we have the following table.

\begin{tabular}{|l||c|c|}
\hline & $\leq_{\mathbf{v}}^{Q}$-maximality & Exactness \\
\hline \hline$M C R[3]$ & YES & NO \\
\hline$M C P R[3]$ & NO & YES \\
\hline$E L P R[1]$ & YES & NO \\
\hline$M P R$ & YES & YES \\
\hline
\end{tabular}

\section{Computing the Maximal Partial Rewriting}

To this end, we will first give a characterization of the maximal partial $M$ rewriting of a language $L$. The construction in the proof of our characterization provides the basic algorithm for computing the rewriting $M P R_{M}(L)$ on a give regular language $L$.

The construction is based on finite transducers. A finite transducer $T=$ $\left(S, I, O, \delta, s_{0}, F\right)$ consists of a finite set of states $S$, an input alphabet $I$, and an output alphabet $O$, a starting state $s_{0}$, a set of final states $F$, and a transitionoutput relation $\delta \subseteq S \times I^{*} \times S \times O^{*}$. Intuitively, for instance $\left(s_{0}, U, s_{1}, W\right) \in$ $\delta$ means that if the transducer is in state $s_{0}$ and reads word $U$ it can go to state $s_{1}$ and emit the word $W$. For a given word $U \in I^{*}$, we say that a word $W \in O^{*}$ is an output of $T$ for $U$ if there exists a sequence $\left(s_{0}, U_{1}, s_{1}, W_{1}\right) \in \delta$, $\left(s_{1}, U_{2}, s_{2}, W_{2}\right) \in \delta, \ldots,\left(s_{n-1}, U_{n}, s_{n}, W_{n}\right) \in \delta$ of state transitions in $T$, such that $s_{n} \in F, U=U_{1} \ldots U_{n}$, and $W=W_{1} \ldots W_{n}$. We write $W \in T(U)$, where $T(U)$ denotes set of all outputs of $T$ for the input word $U$. For a language $L \subseteq I^{*}$ we define $T(L)=\bigcup_{U \in L} T(U)$. It is well known that $T(L)$ is regular whenever $L$ is. 
We are now in a position to state our characterization theorem.

Theorem 4. Let $L$ and $M$ be regular languages over an alphabet $\Delta$. There exists a finite transducer $T$ and a regular language $M^{\prime}$, such that

$$
\operatorname{MPR}_{M}(L)=\left(T\left(L^{c}\right)\right)^{c} \cap M^{\prime},
$$

where $(.)^{c}$ denotes set complement.

Proof. Let $A=\left(S, \Delta, \delta, s_{0}, F\right)$ be a nondeterministic finite automaton that accepts the language $M$. Let us consider the finite transducer:

$$
T=\left(S \cup\left\{s_{0}^{\prime}\right\}, \Delta, \Delta \cup\{m\}, \delta^{\prime}, s_{0}^{\prime},\left\{s_{0}^{\prime}\right\}\right),
$$

where $\delta^{\prime}$, written as a relation, is

$$
\begin{aligned}
\delta^{\prime}= & \left\{\left(s, R, s^{\prime}, \epsilon\right):\left(s, R, s^{\prime}\right) \in \delta\right\} \cup \\
& \left\{\left(s_{0}^{\prime}, R, s_{0}^{\prime}, R\right): R \in \Delta\right\} \cup \\
& \left\{\left(s_{0}^{\prime}, R, s, \epsilon\right):\left(s_{0}, R, s\right) \in \delta\right\} \cup \\
& \left\{\left(s_{0}^{\prime}, R, s_{0}^{\prime}, m\right):\left(s_{0}, R, s\right) \in \delta \text { and } s \in F\right\} \cup \\
& \left\{\left(s, R, s_{0}^{\prime}, m\right):\left(s, R, s^{\prime}\right) \in \delta \text { and } s^{\prime} \in F\right\} .
\end{aligned}
$$

Intuitively, transitions in the first set of $\delta^{\prime}$ are the transitions of the "old" automaton, modified so as to produce $\epsilon$ as output. Transitions in the second set mean that "if we like, we can leave everything unchanged," i.e. each symbol gives itself as output. Transitions in the third set are for jumping non-deterministically from the new initial state $s_{0}^{\prime}$ to the states of the old automaton $A$, that are reachable in one step from the old initial state $s_{0}$. These transitions give $\epsilon$ as output. Transitions in the fourth set are for handling special cases, when from the old initial state $s_{0}$, an old final state can be reached in one step. In these cases we can replace the one symbol words accepted by $A$ with the special symbol $m$. Finally, the transitions of the fifth set are the most significant. Their meaning is: in a state, where the old automaton has a transition by a symbol, say $R$, to an old final state, there will be, in the transducer, an additional transition $R / m$ to $s_{0}^{\prime}$, which is also the (only) final state of $T$. Observe that, if the transducer $T$ decides to leave the state $s_{0}^{\prime}$ while a suffix $U$ of the input string is unscanned, and enter the old automaton $A$, then it can return back only if there is a prefix $U^{\prime}$ of $U$, such that $U^{\prime} \in L(A)$. In such a case, the transducer $T$ replaces $U^{\prime}$ with the special symbol $m$.

Given a word of $W \in \Delta^{*}$ as input, the finite transducer $T$ replaces arbitrarily many occurrences of words of $M$ in $W$ with the special symbol $m$. For an example, suppose $M$ is given by the automaton in Figure 1, top. The corresponding finite transducer is shown in the same figure, bottom. It consists of the automaton for $M$, whose transitions now produce as output $\epsilon$, plus the state $s_{0}^{\prime}$, and the additional transitions, which are drawn with dashed arrows.

If $L^{\prime}$ is a language on $\Delta$, it is straightforward to verify that

$$
\begin{gathered}
T\left(L^{\prime}\right)=L^{\prime} \cup\left\{U_{1} m U_{2} m \ldots m U_{k}:\right. \\
\text { for some } U \in L^{\prime} \text { and words } W_{i} \in M, \\
\left.U=U_{1} W_{1} U_{2} W_{2} \ldots W_{k-1} U_{k}\right\} .
\end{gathered}
$$



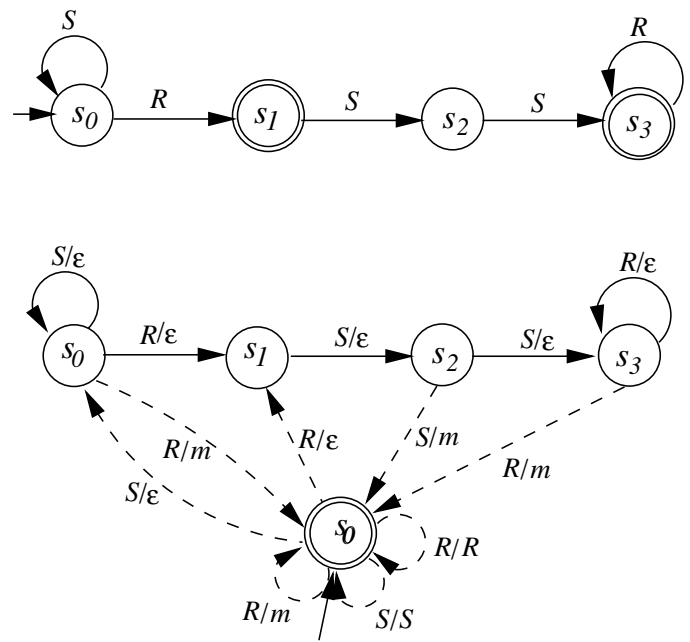

Fig. 1. An example of the construction for a replacement transducer

Recall that, we have $\operatorname{def}(m)=M$, and $\operatorname{def}(R)=R$ for each $R \in \Delta$. From the above, we can also easily characterize the set $T\left(L^{\prime}\right)$ from another point of view. Namely, $T\left(L^{\prime}\right)$ is the set of all words $W$ on $\Delta \cup\{m\}$, such that $\operatorname{def}(W) \cap L^{\prime} \neq \emptyset$.

Now, let's consider the transduction $T\left(L^{c}\right)$. As characterized above, $T\left(L^{c}\right)$ will be the set of all words $W$ on $\Delta \cup\{m\}$ such that $\operatorname{def}(W) \cap L^{c} \neq \emptyset$. Hence, $\left.T\left(L^{c}\right)\right)^{c}$, being the complement of this set, will contain all the $\Delta \cup\{m\}$ words, such that all the $\Delta$-words in their substitution by def will be contained in $L$. This is the containment condition for a word on $\Delta \cup\{m\}$ to be in a rewriting. Clearly, $\left.T\left(L^{c}\right)\right)^{c}$ is a rewriting, and namely, it is the union of all the $M$-rewritings of $L$. Hence, $\left.M P R_{M}(L) \subseteq T\left(L^{c}\right)\right)^{c}$.

However, in order to compute $\operatorname{MPR}_{M}(L)$, what we like is not a contained arbitrary, but a contained exhaustive replacement of words from $M$. To achieve this goal, we should filter out from the set $T\left(L^{c}\right)^{c}$, the words having eligible subwords for replacements (that do not violate the containment constraint). For this, consider the set $(\Delta \cup\{m\})^{*} M(\Delta \cup\{m\})^{*}$. This is the set of all the words on $\Delta \cup\{m\}$ that have at least one potentially eligible subword for replacement. But, as said before, this replacement should be "contained" in the language $L$. Formally speaking, we are interested in solving the following language equation. Find the biggest languages $X, Y \subseteq(\Delta \cup\{m\})^{*}$ such that

$$
X M Y \subseteq\left(T\left(L^{c}\right)\right)^{c} .
$$

Then, if we are able to find such $X$ and $Y$, we have that $X M Y$ is the set of all the words on $\Delta \cup\{m\}$, that still have eligible subwords for replacement. Clearly, we filter out such words and have that

$$
\operatorname{MPR}_{M}(L)=\left(T\left(L^{c}\right)\right)^{c} \cap(X M Y)^{c} .
$$


So, let's examine how to solve the above language equation. For this, consider another special symbol $m^{\prime}$, such that $m^{\prime} \notin \Delta \cup\{m\}$, and the substitution $d e f^{\prime}$ : $\Delta \cup\{m\} \cup\left\{m^{\prime}\right\} \longrightarrow \Delta \cup\{m\}$, such that

$$
d e f^{\prime}\left(m^{\prime}\right)=M, \quad d e f^{\prime}(m)=m, \text { and } \operatorname{def}^{\prime}(R)=R \text { for } R \in \Delta .
$$

Now, we build a transducer $T^{\prime}$ in the same way as we did for the transducer $T$, but for the extended alphabet $\Delta \cup\{m\}$ and considering $m^{\prime}$ as the special symbol. Reasoning similarly as before, if we transduce the complement of $\left(T\left(L^{c}\right)\right)^{c}$ i.e. $T\left(L^{c}\right)$, we have that $\left(T^{\prime}\left(T\left(L^{c}\right)\right)\right)^{c}$ is the set of all words $W \in \Delta \cup\{m\} \cup\left\{m^{\prime}\right\}$, such that $d e f^{\prime}(W) \subseteq\left(T\left(L^{c}\right)\right)^{c}$. However, in order to solve the above language equation, we will be interested in the subset of words in $\left(T^{\prime}\left(T\left(L^{c}\right)\right)\right)^{c}$ that contain exactly one special symbol $\mathrm{m}^{\prime}$. To get this subset, we filter out the un-wanted words by intersecting as in the following

$$
\left(T^{\prime}\left(T\left(L^{c}\right)\right)\right)^{c} \cap(\Delta \cup\{m\})^{*} m^{\prime}(\Delta \cup\{m\})^{*} .
$$

The above is the set of all words $W=W_{1} m^{\prime} W_{2}$, where $W_{1}, W_{2} \in(\Delta \cup\{m\})^{*}$, and $d e f^{\prime}(W) \subseteq\left(T\left(L^{c}\right)\right)^{c}$. Consider now the transducers $T_{l}$ and $T_{r}$ that erase from a word $W \in(\Delta \cup\{m\})^{*} m^{\prime}(\Delta \cup\{m\})^{*}$ the subword in $(\Delta \cup\{m\})^{*} m^{\prime}$ and $m^{\prime}(\Delta \cup\{m\})^{*}$ respectively. Finally, we set

$$
X=T_{r}\left(\left(T^{\prime}\left(T\left(L^{c}\right)\right)\right)^{c} \cap(\Delta \cup\{m\})^{*} m^{\prime}(\Delta \cup\{m\})^{*}\right),
$$

and

$$
Y=T_{l}\left(\left(T^{\prime}\left(T\left(L^{c}\right)\right)\right)^{c} \cap(\Delta \cup\{m\})^{*} m^{\prime}(\Delta \cup\{m\})^{*}\right) .
$$

It is easy to see now that $X$ and $Y$ are regular languages and the maximal solution to the above equation.

As a generalization of Theorem 4 we can give the following result about the maximal partial $\mathbf{V}$-rewriting, of a query $Q$ with respect to a set $\mathbf{V}=\left\{V_{1}, \ldots, V_{n}\right\}$ of view definitions.

Theorem 5. Given a regular path query $Q$, the $M P R_{\mathbf{V}}(Q)$ can be effectively computed.

\section{Optimizing Regular Path Queries Using Partial Rewritings}

In this section we show how to utilize partial rewritings in query optimization in a scenario where we have available a set of precomputed views, as well as the database itself. The views could be materialized views in a warehouse, or locally cached results from previous queries in a client/server environment. In this scenario, the views are assumed to be exact, and we are interested in answering the query by consulting the views as far as possible, and by accessing the database only when necessary. 
Formally, let $\mathbf{V}=\left\{V_{1}, \ldots, V_{n}\right\}$ be a set of view definitions, and let $\Omega=$ $\left\{v_{1}, \ldots, v_{n}\right\}$ be the view alphabet as before. We define the view-graph $\mathcal{V}$ to be a database over $(D, \Omega)$ induced by the set

$$
\bigcup_{i \in\{1, \ldots, n\}}\left\{\left(a, v_{i}, b\right):(a, b) \in \operatorname{ans}\left(V_{i}, D B\right)\right\} .
$$

of $\Omega$-labeled edges.

It has been shown in 4 that, given a query $Q$, if the $M C R_{\mathbf{V}}(Q)$ is exact, then ans $(Q, D B)=\operatorname{ans}\left(M C R_{\mathbf{V}}(Q), \mathcal{V}\right)$. However, the cases when we are able to obtain an exact complete rewriting of the query using the views could be rare in practice. In general, we have in the views only part of the information needed to answer the query. In the following, we will use exact partial rewritings not to completely avoid accessing the database, but to minimize such access as much as possible. We can use an exact partial rewriting $Q^{\prime}$ to evaluate the query on the view-graph, accessing the database in a "lazy" fashion only when necessary.

The intuition behind our algorithm is that we use the view-graph as it was the database, and we extend it "on demand" as the navigation, guided by an automaton for $Q^{\prime}$, requires. In fact, if we need to access a node in the database, we add to the view graph all (not only what we need) the outgoing edges and the neighboring nodes. This was motivated by considering the database as a set of HTML (or XML) pages related to each other through links. Accessing a page in the web could be time consuming, but subsequently, parsing the page text in the main memory for finding the links and the addresses, where these links point, is efficient. Since our queries could be recursive, a page (node) could be visited many times, and if it has already been fetched before from the database, along with all of its links, then there is no need to consult the database again. So, during the execution of the algorithm, for each page $x$ that we fetch from the database, we create a flag Expanded Ex $_{x}$ and set it to true, meaning that now we have full local information for this page.

\section{Algorithm 1}

Input: An exact partial rewriting $Q^{\prime}$ for a query $Q$ and a database $D B$. Output: The answer to the query $\mathrm{Q}$ on database $D B$.

Method: First construct an automaton $A_{Q^{\prime}}$ for $Q^{\prime}$. Let $s_{0}$ be the initial state in $A_{Q^{\prime}}$. Then, for each node $a \in N$, we compute a set Reach ${ }_{a}$ as follows.

1. Initialize Reach $_{a}$ to $\left\{\left(a, s_{0}\right)\right\}$.

2. For each transition $s_{0} \stackrel{R}{\longrightarrow} s$ in $A_{Q^{\prime}}$, access $D B$ and add to $\mathcal{V}$ the subgraph of $D B$ induced by all the edges originating from the nodes $b$, which have some outgoing edges $R$. For each such node $b$, create a flag Expanded Ex $_{b}$ and set it to true.

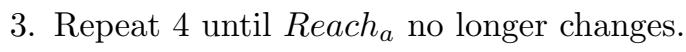

4. Choose a pair $(b, s) \in$ Reach $_{a}$.

a) If there is a transition $s \stackrel{v_{i}}{\longrightarrow} s^{\prime}$ in $A_{Q^{\prime}}$, and there is an edge $b \stackrel{v_{i}}{\longrightarrow} b^{\prime}$ in $\mathcal{V}$, then add the pair $\left(b^{\prime}, s^{\prime}\right)$ to Reach R $^{\prime}$ 
b) Similarly, if there is a transition $s \stackrel{R}{\longrightarrow} s^{\prime}$ in $A_{Q^{\prime}}$, and there is an edge $b \stackrel{R}{\longrightarrow} b^{\prime}$ in $\mathcal{V}$, then add the pair $\left(b^{\prime}, s^{\prime}\right)$ to Reach $_{a}$.

c) Otherwise, if there is a transition $s \stackrel{R}{\longrightarrow} s^{\prime}$ in $A_{Q^{\prime}}$, but there is not an edge $b \stackrel{R}{\longrightarrow} b^{\prime}$ in $\mathcal{V}$, and there is not a flag Expanded $_{b}=$ true, then access the database and add to $\mathcal{V}$ the subgraph of $D B$ induced by all the edges originating from $b$. Create a flag Expanded $_{b}$, and set it to true.

Set $\operatorname{eval}\left(Q^{\prime}, \mathcal{V}, D B\right)=\left\{(a, b):(b, s) \in \operatorname{Reach}_{a}\right.$, and $s$ is a final state in $\left.A_{Q^{\prime}}\right\}$.

It is easy to see that the following theorem is true.

Theorem 6. Given a query $Q$ and a set $\mathcal{V}$ of exact cached views, using an exact partial rewriting $Q^{\prime}$, we have that

$$
\operatorname{eval}\left(Q^{\prime}, \mathcal{V}, D B\right)=\operatorname{ans}(Q, D B) .
$$

\section{Optimizing Conjunctive Regular Path Queries}

A conjunctive regular path query (CRPQ) $Q$ is an expression of the form

$$
Q\left(x_{1}, \ldots, x_{l}\right):-y_{1} E_{1} z_{1}, \ldots, y_{k} E_{k} z_{k}
$$

where $x_{1}, \ldots, x_{l}, y_{1}, \ldots, y_{k}, z_{1}, \ldots, z_{k}$ are (not necessarily all distinct) variables over the universe of objects $D$, such that all the distinguished (head) variables $x_{1}, \ldots, x_{l}$ occur in the body, i. e. each $x_{i}$ is some $y$ or $z$, and $E_{1}, \ldots, E_{k}$ are regular languages (or regular path queries, RPQ) over the database alphabet $\Delta$. We call the conjunctions $y E z$ of a CRPQ, regular path atoms, or simply atoms.

The answer set ans $(Q, D B)$ to a CRPQ Q over a database $D B=(N, E)$ is the set of tuples $\left(a_{1}, \ldots, a_{l}\right)$ of nodes of DB, such that there is a total mapping $\tau$ from $y_{1}, \ldots, y_{k}, z_{1}, \ldots, z_{k}$ to $N$ with $\tau\left(x_{i}\right)=a_{i}$ for every distinguished variable $x_{i}$ of $\mathrm{Q}$, and $(\tau(y), \tau(z)) \in \operatorname{ans}(E, D B)$ for every atom $y E z$ in $Q$.

We say for an atom $y E z$, when $\tau(y)=a$ and $\tau(z)=b$, that $y$ and $z$ have been bound to the objects $a$ and $b$ respectively.

Suppose now, that we have available a set $\mathbf{V}=\left\{V_{1}, \ldots, V_{n}\right\}$ of conjunctive regular path views, which are defined as

$$
V_{i}\left(x_{i 1}, \ldots, x_{i l_{i}}\right):-y_{i 1} E_{i 1} z_{i 1}, \ldots, y_{i k_{i}} E_{i k_{i}} z_{i k_{i}},
$$

for $i \in[1, n]$. Let $\mathbf{E}$ be the set of all $E_{i j}$ above, for $i \in[1, n]$, and $\Omega$ be a set of $e_{i j} \notin \Delta$ corresponding symbols. Then, we define the conjunctive exact partial $\mathbf{V}$ rewriting, or $C E P R_{\mathbf{V}}(Q)$, of a $C R P Q Q$ with respect to a set $\mathbf{V}$ of conjunctive regular path view definitions, as

$$
C E P R_{\mathbf{V}}\left(x_{1}, \ldots, x_{l}\right):-y_{1} E_{1}^{\prime} z_{1}, \ldots, y_{k} E_{k}^{\prime} z_{k}
$$

where $E_{i}^{\prime}$, for $i \in[1, k]$, is an exact partial rewriting of the regular language $E_{i}$ with respect to $\mathbf{E}$, with $\Omega$ as the corresponding set of special symbols. 
Suppose that the sub-mechanism for answering the regular path atoms of the CRPQ's remembers the regular expressions and caches the corresponding answer sets of the regular path atoms in the recently processed CRPQ's. Observe that the cached answer sets of the regular path atoms $E_{i j}$ do not necessarily contain the full set ans $\left(E_{i j}, D B\right)$, but only those pairs that were called for, by the sideways information passing mechanism used. To formalize the "fullness" of a cached answer set, we introduce the notions of the "global completeness" and "local completeness," for the subsets of ans $(E, D B)$, for some $E$.

Let $P$ be a subset of ans $(E, D B)$, for some $E$ and $D B$. Then $P$ is said to be globally complete if $P=\operatorname{ans}(E, D B)$. On the other hand, $P$ is locally complete with respect to a node $a$, if $P \supseteq \sigma_{\$ 1=a}(\operatorname{ans}(E, D B))$. If $P$ is locally complete wrt all nodes in $\pi_{\$ 1}(P)$, we say that $P$ is locally complet 2 .

We observe that, if the query processor can traverse the database graph only in the forward direction (as is the case in the web, see [2]), then the cached answer set of any atom $y E_{i j} z$, for which $z$ is not already bound to some objects, is locally complete. For simplicity, we call the atoms locally complete, when we have computed for them locally complete answer sets. As we will see, the locally complete atoms can be very useful in the query optimization.

Now, let's consider the atoms, which have the $z$ variable already bound to some objects. We observe that, for such atoms, we could have computed locally incomplete answer sets. In order to see this, suppose for example, that the variable $z$ has been bound to the objects $b$ and $c$. Then, if the variable $y$ bounds to an object, say $a$, the RPQ answering sub-mechanism using "cut"-like constructs, can stop the evaluation after computing in the answer, starting to navigate from $a$, the objects $b$ and $c$. But, the object $a$ could be connected with paths spelling words in the same regular language, to other database objects besides $b$ and $c$.

However, if the variable $z$ has been also bound to another object, say $d$, which cannot be reached from $a$, by following a path spelling a word in $E_{i j}$, then we inevitably have to compute a locally complete answer set for the (sub)atom $a E_{i j} z$, in order to reject the pair $(a, d)$. I such a case, the "cut"-like constructs do not have have a chance to execute. So, $a E_{i j} z$ is locally complete, and we cache its answer set for future optimizations.

Now, let's see how we can optimize the answering of the conjunctive regular path queries using conjunctive exact partial rewritings.

Consider the regular path atom $y E_{i j} z$ in some recently processed view $V_{i}$. As explained before, if the variable $z$ is not bound to some database object, then $y E_{i j} z$ is locally complete. Otherwise, we consider all the (sub)atoms $a E_{i j} z$, which are locally complete. Let's denote with $c^{a c h} e_{i j}$, the cached answer set of the locally complete atom $y E_{i j} z$, or if otherwise, the union of the cached answer sets of its locally complete (sub)atoms. Then, we define the atom-view-graph $\mathcal{V}$ to be a database over $(D, \Omega)$ induced by the set

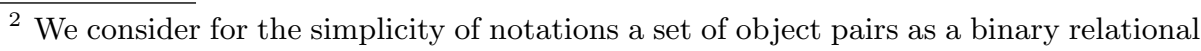
table.
} 


$$
\bigcup_{i \in\{1, \ldots, n\}}\left\{\left(a, e_{i j}, b\right):(a, b) \in \text { cach }_{i j}\right\} .
$$

of $\Omega$-labeled edges 3

The evaluation will alternate between the database-graph and atom-viewgraph. Suppose that we want to answer the atom $y E z$ and $y$ has already been bound to a node, say $a$. We now need to compute the nodes reachable from $a$, by a path spelling a word in $E$. Recall that for $E$ we have computed a corresponding exact rewriting $E^{\prime}$. We start by answering $a E^{\prime} z$ on $D B$. Intuitively, when during the navigation we are in a node $b$, and the regular expression for $E^{\prime}$ requires an $\Omega$-symbol, say $e_{i j}$, to be matched, then we look at the atom-view-graph $\mathcal{V}$. If there is a node $b$ in $\mathcal{V}$, and in that node we find outgoing $e_{i j}$ edges, then we advance the navigation in the atom-view-graph. Since the cached answer for $E_{i j}$ is locally complete, we are sure that we get all the answers had we answered $E_{i j}$ directly in the database starting from $b$.

On the other hand, if we do not find an object $b$ in $\mathcal{V}$, or even if we find $b$ in $\mathcal{V}$, but there are no outgoing edges labeled with $e_{i j}$, then we evaluate $E_{i j}$, starting from $b$ in the database graph, and enrich the atom-view-graph accordingly. Clearly, we do not add by this operation any overhead for answering parts of some new $\Delta^{*}$ word, that is not in $E$, because the rewriting is exact. Formally, we have the following algorithm for answering $a E z$ on the basis of $E^{\prime}$.

\section{Algorithm 2}

Input: An exact rewriting $E^{\prime}$ for the (sub)atom $a E z$, and a database $D B$.

Output: The answer to the (sub)atom $a E z$ on database $D B$.

Method: First construct an automaton $A_{E^{\prime}}$ for $E^{\prime}$. Let $s_{0}$ be the initial state in $A_{E^{\prime}}$. We compute the set Reach ${ }_{a}$ as follows.

1. Initialize Reach to $\left._{a}\left(a, s_{0}\right)\right\}$.

2. Repeat 3 until Reach $a$ no longer changes.

3. Choose a pair $(b, s) \in$ Reach $_{a}$.

a) If there is a transition $s \stackrel{e_{i j}}{\longrightarrow} s^{\prime}$ in $A_{E^{\prime}}$, and there is an edge $b \stackrel{e_{i j}}{\longrightarrow} b^{\prime}$ in $\mathcal{V}$, then add the pair $\left(b^{\prime}, s^{\prime}\right)$ to Reach $_{a}$.

b) Otherwise, if there is a transition $s \stackrel{e_{i j}}{\longrightarrow} s^{\prime}$ in $A_{E^{\prime}}$, but there is not an edge $b \stackrel{e_{i j}}{\longrightarrow} b^{\prime}$ in $\mathcal{V}$, answer $b E_{i j} z$ on $D B$ and enrich $\mathcal{V}$ accordingly.

c) If there is a transition $s \stackrel{R}{\longrightarrow} s^{\prime}$ in $A_{E^{\prime}}$, and there is an edge $b \stackrel{R}{\longrightarrow} b^{\prime}$ in the database $D B$, then add the pair $\left(b^{\prime}, s^{\prime}\right)$ to Reach $_{a}$.

Finally, set $\operatorname{eval}\left(E^{\prime}, a, D B\right)=\left\{(a, b):(b, s) \in\right.$ Reach $_{a}$, and $s$ is a final state in $A\}$.

It is easy to see that the following theorem is true.

\footnotetext{
${ }^{3}$ From another point of view, we can also see that a regular path atom (or (sub)atom), along with its locally complete answer set, is nothing else but a local node constraint as defined in 2 .
} 
Theorem 7. Given a CRPQ $Q$ and a set $\mathbf{V}$ of views as above, using the rewriting $C E P R_{\mathbf{V}}(Q)$, we have that for some regular atom $E$ in $Q$, the answer to aEz equals $\operatorname{eval}\left(E^{\prime}, a, D B\right)$.

Now, for the case of regular path atoms $a E z$, where the variable $z$ has already been bound to some objects, we slightly modify the above algorithm to evaluate the answer to such atoms as well. For this, let $B$ be the set of objects where $z$ has been bound. Then, in the above algorithm we incrementally compute in each step the set $\operatorname{eval}\left(E^{\prime}, a, D B\right)$ and change the loop (2) to

Repeat 3 until Reach $a$ no longer changes or $\pi_{\$ 2}\left(\operatorname{eval}\left(E^{\prime}, a, D B\right)\right)$ equals $B$.

We note that, the second condition of the loop termination is there for restricting the search space, in case we find (or verify) that we can reach from $a$ all the objects in $B$ by following paths, which spell words in $E$. Clearly, in this case, the answer set of the atom $a E z$ equals $a \times B$. On the other hand, we also stop if $R_{e a c h}$ reaches a fixed point, and in this case the answer to the atom $a E z$ equals $a \times\left(\pi_{\$ 2}\left(\operatorname{eval}\left(E^{\prime}, a, D B\right)\right) \cap B\right)$. In such a case, although the variable $z$ had already been bound, the set $\left.\operatorname{eval}\left(E^{\prime}, a, D B\right)\right)$ is fully computed and so, it is locally complete.

Finally, if we set $B=\emptyset$, when the variable $z$ has not been already bound to some objects, then we can have a single $R P Q$-answer-and-cache algorithm. This algorithm would compute the answer set to an atom $y E z$, by iterating over all the objects $a$, where the variable $y$ could be bound, and compute $a E z$ by using an exact partial rewriting $a E^{\prime} z$, as described above. At the end, we check whether the set Reach $_{a}$ has reached a fixed point. If yes, then we cache the locally complete $\left.\operatorname{eval}\left(E^{\prime}, a, D B\right)\right)$, for future RPQ optimizations.

\section{Complexity Analysis}

Theorem 8. Given a language $L$ and an $\epsilon$-free language $M$ both of them over an alphabet $\Delta$, the problem of generating the $M P R_{M}(L)$ is in $3 E X P T I M E$.

From the above theorem, we can easily derive the following corollary, regarding the upper complexity bound for the generation of the $M P R_{\mathbf{V}}(Q)$ of a query $Q$, with respect to a set $\mathbf{V}=\left\{V_{1}, \ldots, V_{n}\right\}$ of view definitions.

Corollary 3. Given a language $Q$ and a set $\mathbf{V}=\left\{V_{1}, \ldots, V_{n}\right\}$ of view definitions, the problem of generating the $M P R_{\mathbf{V}}(Q)$ is in $3 E X P T I M E$.

We emphasize here that the above complexity analysis is a worst case analysis. In fact, the above complexity comes from possible DFA's state "blow up." However, despite these worst case possibilities, experimental results in 88, for converting graphs into DFA's, are encouraging, indicating that for the majority of the cases, the running time is very reasonable and the resulting DFA's are significantly smaller than their sources. Also, observe that if the probability of getting an exponential size DFA for an NFA is $p<<1$, then the probability of getting a triple exponential "blow up" in our case would roughly be $p^{3}$, which is very small. 
Now, in order to prove that the above established upper bound is essentially optimal we will need the following constructions and theorems. Consider the set

$$
\left(T\left(Q^{c}\right)\right)^{c} \cap\left((\Delta \cup \Omega)^{*}\left(V_{1} \cup \ldots \cup V_{n}\right)(\Delta \cup \Omega)^{*}\right)^{c},
$$

where the transducer $T$ is defined as in Theorem [5. This is in essence the $e x$ haustive lower partial rewriting, or $\operatorname{ELPR}_{\mathbf{V}}(Q)$ of [11], which is also discussed in Section 3. It is the set of all "mixed" words $W$ on the alphabet $\Omega \cup \Delta$, with no subword in $V_{1} \cup \cdots \cup V_{n}$, such that their substitution by def is contained in the query $Q$. Obviously, $\operatorname{def}\left(\operatorname{ELPR}_{\mathbf{V}}(Q)\right) \subseteq Q$ and we are interested in the complexity of testing its exactness with respect to the query $Q 4$. We prove the following theorem regarding the upper bound of the above exactness problem.

Theorem 9. Given a query $Q$ and a set $\mathbf{V}=\left\{V_{1}, \ldots, V_{n}\right\}$ of view definitions, the problem of testing $\operatorname{def}\left(E L P R_{\mathbf{V}}(Q)\right)=Q$ is in 2EXPSPACE.

Proof. By Theorem 8 the automaton $\left(T\left(Q^{c}\right)\right)^{c}$ can exponentially "blow up" and namely be of doubly exponential size. The automaton for

$$
\left((\Delta \cup \Omega)^{*}\left(V_{1} \cup \ldots \cup V_{n}\right)(\Delta \cup \Omega)^{*}\right)^{c}
$$

can also exponentially "blow up" but its size can be up to single exponential. So, in total the automaton for $E L P R_{\mathbf{V}}(Q)$ can be of up to doubly exponential size. Now, let's consider $\operatorname{def}\left(E L P R_{\mathbf{V}}(Q)\right)$. For the substitution def there exists a polynomial size transducer $T_{\text {def }}$ such that $\operatorname{def}\left(\operatorname{ELP} R_{\mathbf{V}}(Q)\right)=$ $T_{\text {def }}\left(E L P R_{\mathbf{V}}(Q)\right)\left[1910\right.$. Thus, the size of the automaton $T_{\text {def }}\left(E L P R_{\mathbf{V}}(Q)\right)$ will be polynomial on the size of the automaton for $E L P R_{\mathbf{V}}(Q)$ i.e. it can be doubly exponential on the size of the automaton for $Q$. Now, to test the exactness $\operatorname{def}\left(E L P R_{\mathbf{V}}(Q)\right)=Q$ is in PSPACE with regard to the size of the automaton for $E L P R_{\mathbf{V}}(Q)$. So, in total we have that testing the exactness of $E L P R_{\mathbf{V}}(Q)$ is in 2EXPSPACE.

For the lower bound of the exactness $\operatorname{def}\left(\operatorname{ELPR}_{\mathbf{V}}(Q)\right)=Q$ we will use the maximally contained rewriting, $M C R_{\mathbf{V}}(Q)$ of [3]. As mentioned in Section[2, the $M C R_{\mathbf{V}}(Q)$ is the set of all words $W$ in $\Omega^{*}$, such that $\operatorname{def}(W) \in Q$. In [3], the exactness problem for the $M C R_{\mathbf{V}}(Q)$ is proven to be 2EXPSPACE complete. We will give in the following a reduction of the exactness problem for $M C R_{\mathbf{V}}(Q)$ to the exactness problem for $\operatorname{ELP} R_{\mathbf{V}}(Q)$.

Consider a query $Q$ and a set $\mathbf{V}=\left\{V_{1}, \ldots, V_{n}\right\}$ of view definitions. Now, consider the set $\mathbf{V}^{\prime}=\left\{V_{1}^{\prime}, \ldots, V_{n}^{\prime}\right\}$ of new view definitions, where $V_{i}^{\prime}=\$ V_{i} \$$ for $i=[1, n]$ and $\$ \notin \Delta \cup \Omega$. Regarding the query $Q$, we will transform it into a new query $Q^{\prime}$ through a transduction. For this we construct the following transducer.

Let $A_{i}=\left(S_{i}, \Delta, \delta_{i}, s_{0 i}, F_{i}\right)$, for $i \in[1, n]$ be $n$ nondeterministic finite automata that accept the corresponding $V_{i}$ languages. Let us consider the finite transducer: $T_{\$ \$}=\left(S_{1} \cup \ldots \cup S_{n} \cup\left\{s_{0}^{\prime}\right\}, \Delta, \Delta \cup\{\$\}, \delta^{\prime}, s_{0}^{\prime},\left\{s_{0}^{\prime}\right\}\right)$, where

$$
\delta^{\prime}=\left\{\left(s, R, s^{\prime}, R\right):\left(s, R, s^{\prime}\right) \in \delta_{i}, i \in[1, n]\right\} \cup
$$

$\overline{4}$ The complexity bounds for testing the exactness $E L P R_{\mathbf{V}}(Q)$ are not discussed in 11. 


$$
\begin{aligned}
& \left\{\left(s_{0}^{\prime}, \epsilon, s_{0 i}, \$\right): i \in[1, n]\right\} \cup \\
& \left\{\left(s, \epsilon, s_{0}^{\prime}, \$\right): s \in F_{i}, i \in[1, n]\right\} .
\end{aligned}
$$

The transducer $T_{\$ \$}$ performs the following task: given a language $L$ as input, it produces as output all the words of $L$ having inserted in them the symbol $\$$ to mark the beginning and the end of a subword that belongs to $V_{i}$ for some $i \in[1, n]$. Moreover, only the words of $L$, which we can divide into a sequence of subwords, such that each belongs to some view language, are transduced. All the other $L$ words are filtered out. Formally,

$$
\begin{aligned}
T_{\$ \$}(L)= & \left\{\$ U_{1} \$ \$ U_{2} \$ \ldots \$ U_{k} \$:\right. \\
& \text { for some } U \text { in } L, U=U_{1} U_{2} \ldots U_{k} \\
& \text { and } \left.\forall i \in[1, k] \exists j \in[1, n] \text { such that } U_{i} \in V_{j}\right\} .
\end{aligned}
$$

Theorem 10. Consider a query $Q$ and a set $\mathbf{V}=\left\{V_{1}, \ldots, V_{n}\right\}$ of view definitions. Construct $Q$ and $\mathbf{V}^{\prime}=\left\{V_{1}^{\prime}, \ldots, V_{n}^{\prime}\right\}$ as above. Let $\Omega^{\prime}=\left\{v_{1}^{\prime}, \ldots, v_{n}^{\prime}\right\}$ and def $f^{\prime}$ be the usual view substitution on $\Omega^{\prime}$, i.e. def $^{\prime}\left(v_{i}^{\prime}\right)=V_{i}^{\prime}$, for $i \in[1, n]$. Also, let def $f_{\$ \rightarrow \epsilon}$ be the substitution that erases the symbol $\$$ from some language on $\Delta \cup\{\$\}$. Then, the following is true: the $M C R_{\mathbf{V}}(Q)$ is exact if and only if

1. $\operatorname{def}_{\$ \rightarrow \epsilon}\left(Q^{\prime}\right)=Q$, and

2. $E L P R_{\mathbf{V}^{\prime}}\left(Q^{\prime}\right)$, with respect to $\mathbf{V}^{\prime}=\left\{V_{1}^{\prime}, \ldots, V_{n}^{\prime}\right\}$, is exact.

Based on the above theorem we give the following theorem regarding the optimality of the construction given in Theorem 9] for testing the exactness of the ELPR of a query $Q$.

Theorem 11. Given a query $Q$ and a set $\mathbf{V}=\left\{V_{1}, \ldots, V_{n}\right\}$ of view definitions, the problem of testing def $\left(E L P R_{\mathbf{V}}(Q)\right)=Q$ is 2EXPSPACE complete.

Finally, the following theorem shows the optimality of our algorithm for computing the MPR of a query using a set of view definitions.

Theorem 12. The algorithm presented in Theorem 5 for computing the rewriting $M P R_{\mathbf{V}}(Q)$ of a query $Q$ given a set $\mathbf{V}=\left\{V_{1}, \ldots, V_{n}\right\}$ of view definitions, is essentially optimal.

Proof. Consider the $E L P R_{\mathbf{V}}(Q)$. Recall that this is the set of all "mixed" words $W$ on the alphabet $\Omega \cup \Delta$, with no subword in $V_{1} \cup \cdots \cup V_{n}$, such that their substitution by def is contained in the query $Q$. So, such words qualify for inclusion in $M P R_{\mathbf{V}}(Q)$. Hence, we have that $\operatorname{ELPR}_{\mathbf{V}}(Q) \subseteq \operatorname{CEPR}(Q)$. On the other hand, observe that if $\operatorname{ELPR}_{\mathbf{V}}(Q)$ is exact then we must have $\operatorname{ELP} R_{\mathbf{V}}(Q)$ $=M P R_{\mathbf{V}}(Q)$. Now, we can test the exactness of $E L P R_{\mathbf{V}}(Q)$ by testing the condition $M P R_{\mathbf{V}}(Q) \subseteq E L P R_{\mathbf{V}}(Q)$, which is equivalent with the non emptiness of $M P R_{\mathbf{V}}(Q) \cap\left(E L P R_{\mathbf{V}}(Q)\right)^{c}$. Since, by Theorem 9] we get a DFA for $E L P R_{\mathbf{V}}(Q)$, to complement does not add anything to the complexity of testing the emptiness of the above intersection. Now, if we were able to generate 
the $M P R_{\mathbf{V}}(Q)$ in, say, 2EXPTIME, and since we are able to generate the $E L P R_{\mathbf{V}}(Q)$ in 2EXPTIME, then we could test the exactness of $E L P R_{\mathbf{V}}(Q)$ in 2EXPTIME, which is impossible by Theorem 11, unless 2EXPTIME = 2EXPSPACE.

\section{References}

1. S. Abiteboul, P. Buneman and D. Suciu. Data on the Web : From Relations to Semistructured Data and XML. Morgan Kaufmann, 1999.

2. S. Abiteboul, V. Vianu. Regular Path Queries with Constraints. Journal of Computing and System Sciences 58(3) 1999, pp. 428-452

3. D. Calvanese, G. Giacomo, M. Lenzerini and M. Y. Vardi. Rewriting of Regular Expressions and Regular Path Queries. Proc. of PODS 1999, pp. 194-204.

4. D. Calvanese, G. Giacomo, M. Lenzerini and M. Y. Vardi. Answering Regular Path Queries Using Views. Proc. of ICDE 2000, pp. 389-398.

5. D. Calvanese, G. Giacomo, M. Lenzerini and M. Y. Vardi. View-Based Query Processing for Regular Path Queries with Inverse. Proc. of PODS 2000, pp. 58-66.

6. D. Calvanese, G. Giacomo, M. Lenzerini and M. Y. Vardi. What is View-Based Query Rewriting? Proc. of KRDB 2000, pp. 17-27.

7. D. Florescu, A. Y. Levy, D. Suciu Query Containment for Conjunctive Queries with Regular Expressions Proc. of PODS 1998, pp. 139-148.

8. R. Goldman and J. Widom. DataGuides: Enabling query Formulation and Optimization in Semistructured Databases. Proc. of VLDB 1997 pp. 436-445.

9. G. Grahne and A. O. Mendelzon. Tableau Techniques for Querying Information Sources through Global Schemas. Proc. of ICDT 1999 pp. 332-347.

10. G. Grahne and A. Thomo. An Optimization Technique for Answering Regular Path Queries. Proc. of WebDB 2000.

11. G. Grahne and A. Thomo. Algebraic Rewritings for Optimizing Regular Path Queries. Proc. of ICDT 2001 pp. 301-315.

12. G. Grahne and A. Thomo. New Rewritings and Optimizations for Regular Path Queries. www.cs.concordia.ca/ ${ }^{\text {faculty/grahne/papers/fullicdt03.ps }}$

13. J. E. Hopcroft and J. D. Ullman Introduction to Automata Theory, Languages, and Computation. Addison-Wesley 1979.

14. L. Kari. On Insertion and Deletion in Formal Languages. Ph.D. Thesis, 1991, Department of Mathematics, University of Turku, Finland.

15. A. Y. Levy. Answering queries using views: a survey. Technical Report, Computer Science Dept., Washington Univ., 2000.

16. A. Y. Levy, A. O. Mendelzon, Y. Sagiv, D. Srivastava. Answering Queries Using Views. Proc. of PODS 1995, pp. 95-104.

17. A. O. Mendelzon and P. T. Wood, Finding Regular Simple Paths in Graph Databases. SIAM J. Comp. 24:6, (December 1995).

18. Y. Papakonstantinou, V. Vassalos. Query Rewriting for Semistructured Data. Proc. of SIGMOD 1999, pp. 455-466

19. S. Yu. Regular Languages. In: Handbook of Formal Languages. G. Rozenberg and A. Salomaa (Eds.). Springer Verlag 1997, pp. 41-110 